\title{
Long Dip-Slip Fault in a Viscoelastic Half Space Model of the Lithosphere
}

\author{
Sanjay Sen", Subrata Kr. Debnath" ${ }^{2, *}$ \\ ${ }^{\mathbf{1}}$ Department of Applied Mathematics, University of Calcutta, 92, APC Road, Kolkata, 700009, India \\ ${ }^{2}$ Department of Basic Science and Humanities, Meghnad Saha Institute of Technology (A unit of Techno India Group), Nazirabad.P.O. \\ Uchhepota, Via- Sonarpur, Kolkata, 700150, India
}

\begin{abstract}
Most of the earthquake faults in North- East India, China, mid Atlantic-ridge, the Pacific seis mic belt and Japan are found to be predominantly dip-slip in nature. In the present paper a dip -slip fault is taken to be situated in a viscoelastic half space representing the upper lithospheric region of the Earth. A movement of the dip -slip nature across the fault occurs when the accumulated stress due to various tectonic reasons e.g. mantle convection etc, exceeds the local friction and cohesive forces across the fault. The movement is assumed to be creeping in nature, expressions for displacement, stress and strains are obtained by suitable mathe matical methods. A detailed study of these expressions may give some ideas about the nature of stress accumulation in the system, which in turn will be helpful in formu lating an earthquake pred iction programme.
\end{abstract}

Keywords Aseismic Period, Dip-Slip Fault, Earthquake Prediction, Mantle Convection, Plate Movements, Stress Accumulation, Tectonic Process, Viscoelastic Half Space

\section{Introduction}

It is the observational fact that while some faults are strike slip (finite or infinite in length) in nature, there are faults (e.g: Sierra Nevada/Owens valley: Basin and Range faults, Rocky Mountains, Himalayas, Atalanti fault of central Greece-a steeply dipping fault with dip 60,80(deg)) where the surface level changes during the motion i.e. the faults are dip-slip in nature.

A pioneering work involving static ground deformation in elastic media were initiated by ([37-38]). ([4-7]), ([15-16]). [27],[34],[35] did a wonderful work in analyzing the displacement, stress and strain for dip-slip movement. Later some theoretical models in this direction have been formulated by a number of authors like[2],[31],[23],[32], [41][24],[22],[33],[11],[3],[29], ([18-21]),[8],[26],[28],[12], [30],[44],[42],[43],[25],[45],[36] has discussed various aspects of fault movement in his book. Reference; [13] has discussed stress accumulation near buried fault inlithosphere-asthenosphere system. The work of[10] can also be mentioned.

In most of these works the medium were taken to be elastic and /or viscoelastic, some authors preferred layered model with elastic layer(s) over elastic or viscoelastic half space.

In the present case we consider a long dip-s lip fault

* Corresponding author:

skd.sxccal@gmail.com (Subrata Kr. Debnath)

Published online at http://journal.sapub.org/ajcam

Copyright (C) 2012 Scientific \& Academic Publishing. All Rights Reserved situated in a viscoelas tic half space which reaching upto the free surface. The medium is taken to be under the influence of tectonic forces due to mantle convection or some related phenomena. The fault is assumed to undergo a creeping movement when the stresses in the region exceed certain threshold values.

In our paper, we consider a viscoelastic half space to represent the upper part of the lithosphere-asthenosphere system, with constant rigidity $\left(2: 0 \times 10^{5} \mathrm{Mpa}\right)$ and viscosity $\left(10^{20}-10^{21}\right.$ pa:s $)$ following the observational data mentioned by[9],[14]. It may be stated that a thin elastic layer overlying an elastic/viscoelastic half space is likely to be a more preferable model for the system. But, numerical computational works indicate that the presence of a layer does not lead to any significant qualitative changes in the nature of the stress and strain accumulation in the model, only a small 10 percent quantitative change were observed. Analytical expressions for displacements stresses and strains are obtained both before and after the fault movement using appropriate mathematical technique involving integral transformation, Green's function. Numerical computational works have been carried out with suitable values of the model parameters and the nature of the stress and strain accumulation in the mediu $m$ have been investigated.

\section{Formulation}

We consider a long dip-slip fault $F$, width D situated in a viscoelastic half space of linear Maxwell type.

A Cartesian coordinate system is used with a suitable 
point $\mathrm{O}$ on the strike of the fau lt as the origin, the strike of the fault along the $Y_{1}$ axis and $Y_{2}$ axis is as shown in Figure 1, and $Y_{3}$ axis pointing downwards. We choose another coordinate system $Y_{1}{ }^{\prime}, Y_{2}{ }^{\prime}$ and $Y_{3}{ }^{\prime}$ axes as shown in Figure. 1 below, so that the fault is given by $F:\left(y_{2}{ }^{\prime}=0,0 \leq y_{3}{ }^{\prime} \leq D\right)$. Let $\theta$ be the dip of the fault $F$.

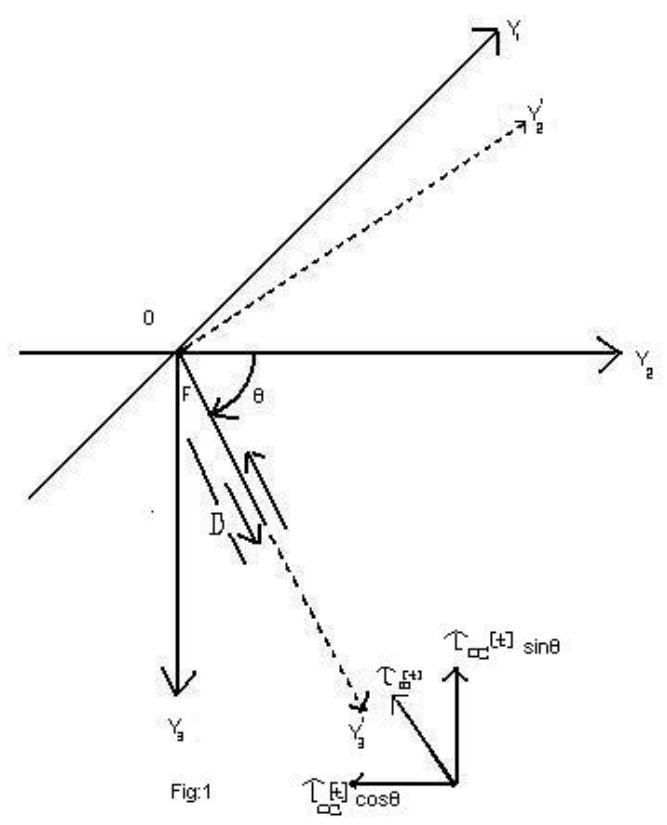

Figure 1. Section of the model by the plane $y_{1}=0$

For a viscoelastic Maxwell type medium the constitutive equations are taken as:

$$
\begin{gathered}
\left(\frac{1}{\eta}+\frac{1}{\mu} \frac{\partial}{\partial t}\right) \tau_{22}=\frac{\partial}{\partial t}\left(e_{22}\right)=\frac{\partial}{\partial t}\left(\frac{\partial v}{\partial y_{2}}\right) \\
\left(\frac{1}{\eta}+\mid \frac{1}{\mu} \frac{\partial}{\partial t}\right) \tau_{23}=\frac{\partial}{\partial t}\left(e_{23}\right)=\frac{1}{2}\left(\frac{\partial v}{\partial y_{3}}+\frac{\partial v}{\partial y_{2}}\right) \\
\left(\frac{1}{\eta}+\frac{1}{\mu} \frac{\partial}{\partial t}\right) \tau_{33}=\frac{\partial}{\partial t}\left(e_{33}\right)=\frac{\partial}{\partial t}\left(\frac{\partial w}{\partial y_{3}}\right)
\end{gathered}
$$

Where $\eta$ is the effective $v$ iscosity and $\mu$ is the effective rigidity of the material.

The stresses satisfy the following equations: (assuming quasistatic deformation for which the inertia terms are neglected).

$$
\begin{aligned}
& \frac{\partial}{\partial y_{1}}\left(\tau_{11}\right) \frac{\partial}{\partial y_{2}}\left(\tau_{12}\right)+\frac{\partial}{\partial y_{3}}\left(\tau_{13}\right)=0 \\
& \frac{\partial}{\partial y_{1}}\left(\tau_{21}\right) \frac{\partial}{\partial y_{2}}\left(\tau_{22}\right)+\frac{\partial}{\partial y_{3}}\left(\tau_{23}\right)=0 \\
& \frac{\partial}{\partial y_{1}}\left(\tau_{31}\right) \frac{\partial}{\partial y_{2}}\left(\tau_{32}\right)+\frac{\partial}{\partial y_{3}}\left(\tau_{33}\right)=0
\end{aligned}
$$

Where $\left(\left(-\infty<y_{2}<\infty\right), y_{3} \geq 0, t \geq 0\right)$ (Assuming the body forces do not change during the fault movement).

The boundary conditions are taken as, with $t=0$ representing an instant when the medium is in aseismic state:

$$
\begin{aligned}
& \tau_{22}\left(y_{2}, y_{3}, t\right)=\tau_{\infty}(t) \cos \theta \text { as } \\
& \left|y_{2}\right| \rightarrow \infty, y_{3} \geq 0, t>0
\end{aligned}
$$

On the free surface

$$
\begin{gathered}
y_{3}=0,\left(-\infty<y_{2}<\infty, t \geq 0\right) \\
\tau_{23}\left(y_{2}, y_{3}, t\right)=0 \\
\tau_{33}\left(y_{2}, y_{3}, t\right)=0 \\
y_{3} \rightarrow \infty\left(-\infty<y_{2}<\infty, t \geq 0\right) \\
\tau_{23}\left(y_{2}, y_{3}, t\right)=0 \\
\tau_{33}\left(y_{2}, y_{3}, t\right)=\tau_{\infty}(t) \sin \theta
\end{gathered}
$$

Also, as $y_{3} \rightarrow \infty\left(-\infty<y_{2}<\infty, t \geq 0\right)$

[Where $\tau_{\infty}(t)$ is the shear stress maintained by mantle convection and other tectonic phenomena far away from the fault].

The initial conditions are: $\operatorname{Let}(v)_{0} ;(w)_{0},\left(\tau_{i j}\right)_{0}$ and $\left(e_{i j}\right)_{0}$ $i, j=2,3$ be the value of $(v) ;(w),\left(\tau_{i j}\right)$ and $\left(e_{i j}\right)$ at time $t=0$ which are functions of $y_{2}, y_{3}$ and satisfy the relations (1.1)-(1.11).

\subsection{Solutions in the Absence of Any Fault Dislocation}

The boundary value problem given by (1.1)-(1.11), can be solved(as shown in the Appendix-1) by taking Laplace transform with respect to time ' $t$ ' of all the constitutive equations and the boundary conditions. On taking the inverse Laplace transform we get the solutions for displacements, stres ses as:

$$
\begin{gathered}
v\left(y_{2}, y_{3}, t\right)=(v)_{0}+(\cos \theta / \mu) \times \\
\times\left[\left(\tau_{\infty}(t)-\tau_{\infty}(0)+\left(\frac{\mu}{\eta}\right) \int_{0}^{t} \tau_{\infty}(\tau) d \tau\right]\right. \\
w\left(y_{2}, y_{3}, t\right)=(w)_{0}+\left(y_{3} / \mu\right) \times \\
\times\left[\left(\tau_{\infty}(t)-\tau_{\infty}(0)+\left(\frac{\mu}{\eta}\right) \int_{0}^{t} \tau_{\infty}(\tau) d \tau\right] \sin \theta\right. \\
\tau_{22}-\tau_{\infty}(t) \cos \theta-\left[\tau_{\infty}(0) \cos \theta-\left(\tau_{22}\right)_{0}\right] e^{-(\mu / \eta) t} \\
\tau_{23}=\left(\tau_{23}\right)_{0} e^{-(\mu / \eta) t} \\
\tau_{33}=\left[\tau_{\infty}(t)-\tau_{\infty}(0) e^{-(\mu / \eta) t}\right] \sin \theta \text { (A) }
\end{gathered}
$$

From the above solution we find that $\tau_{22}$ increases with time and tends to $\tau_{\infty}(t) \cos \theta$ as $t$ tends to $\infty$, while $\tau_{23}$, tends to zero, but $\tau_{33}$ tends to $\tau_{\infty}(t) \sin \theta$. We assume that the geological conditions as well as the characteristic of the fault is such that when the stress-component $\tau_{2^{\prime} 3^{\prime}}$ reaches some critical value, say $\tau_{\mathrm{c}}<\tau(t) \cos \theta$ the fault $F$ starts creeping.

For bounded stress and strains, the creep function should satisfy the following conditions as discussed by Mukhopadhyay et al.(1983,a).

$\left(C_{1}\right)$ Its value will be maximum on the free surface.

$\left(C_{2}\right)$ The magnitude of the creep will decrease with $y_{3}$ as we move down wards and ultimately tends to zero near the lower edge of the fault.

$$
\left(y_{2}^{\prime}=0, y_{3}^{\prime}=D\right)
$$

If $g\left(x_{3}^{\prime}\right)$ be the creep function, it should satisfy the above conditions. 


\subsection{Solutions after the Fault Movements}

We assume that after a time $T_{1}$, the stress component $\tau_{2^{\prime} 3^{\prime}}$ (which is the main driving force for the dip-slip motion of the fault) exceeds the critical value $\tau_{e}$, and the fault $F$ starts creeping, characterized by a dislocation across the fault given by (Appendix-2).

$$
[(w)]_{F}=w_{1}\left(t_{1}\right) g\left(y_{3}\right) H\left(t_{1}\right)
$$

Where, $H\left(t_{1}\right)$ is the Heaviside function and $[(w)]_{F}=$ the discontinuity of $w$ across $F$ given by:

$$
\begin{gathered}
{[(w)]_{F}=\lim _{\left(y_{2}^{\prime} \rightarrow 0+\right)}(w)-\lim _{\left(y_{2}^{\prime} \rightarrow 0-\right)}(w)} \\
\left(y_{2}^{\prime}=0,0 \leq y_{3}^{\prime} \leq D\right)
\end{gathered}
$$

We solve the resulting boundary value problem by modified Green's function method following[16],[31] and correspondence principle (As shown in the Appendix 2) and get the solution for displacements, stresses and strain as :

$$
\begin{gathered}
v\left(y_{2}, y_{1}, \theta, t\right)=(v)_{0}+(\cos \theta / \mu) \\
\left.\left[\tau_{\infty}(0)\right)+(\mu / \eta) \int_{0}^{t} \tau_{\infty}(\tau) d \tau\right] \\
w\left(y_{2}, y_{3}, \theta, t\right)=(w)_{0}+\left(y_{3} / \mu\right) \\
\left.\left[\tau_{\infty}(0)\right)+(\mu / \eta) \int_{0}^{t} \tau_{\infty}(\tau) d \tau\right] \sin \theta \\
+\left[H\left(t-T_{1}\right) /(2 \times \pi)\right] w_{1}\left(t_{1}\right) e^{(-\mu / \eta) t} \int_{0}^{D} g\left(x_{3}^{\prime}\right) \\
{\left[A_{1} / B_{1}+C_{1} / D_{1}\right] d x_{3}^{\prime}}
\end{gathered}
$$

Where

$$
\begin{aligned}
& A_{1}=\left(y_{2}\right) \sin \theta-\left(y_{3}\right) \cos \theta \\
& B_{1}=\left[\left(x_{3}^{\prime 2}\right)-2\left(x_{3}^{\prime}\right)\left(y_{3} \cos \theta+y_{3} \sin \theta\right)+\right. \\
& \left.+\left(y_{3}^{2}\right)+\left(y_{3}^{2}\right)\right] \\
& \tau_{22}\left(y_{2}, y_{3}, \theta, t\right)=\tau_{\infty}(t) \cos \theta-\left(\tau_{\infty}(0) \cos \theta-\right. \\
& \left.-\left(\tau_{22}\right)_{0}\right) e^{(-\mu / \eta) t} \\
& \left.\tau_{23}\left(y_{2}, y_{3}, \theta, t\right)=\left(\tau_{23}\right)_{0}\right) e^{(-\mu / \eta)}+ \\
& \left.+\left[H\left(t-T_{1}\right) /(2 \times \pi)\right] w_{1}\left(t_{1}\right) e^{(-\mu / \eta)}\right] \int_{0}^{D} \\
& g\left(x_{3}^{\prime}\right)\left[A_{2} / B_{2}+C_{2} / D_{2}\right] d x_{3}^{\prime}
\end{aligned}
$$

Where,

$$
\begin{aligned}
& A_{2}=\left(x_{3}^{\prime 2}-2 x_{3}^{\prime}\left(y_{2} \cos \theta+y_{3} \sin \theta\right)+y_{2}^{2}+y_{3}^{2}\right) \\
& \sin \theta-\left(y_{2} \sin \theta-y_{3} \cos \theta\right)\left(2 y_{2}-2 x_{3}^{\prime} \cos \theta\right) \\
& B_{2}=x_{3}^{\prime 2}-2 x_{3}^{\prime}\left(y_{2} \cos \theta+y_{3} \sin \theta\right)+y_{2}^{2}+y_{3}^{2}, \\
& C_{2}=\left(x_{3}^{\prime 2}-2 x_{3}^{\prime}\left(y_{2} \cos \theta-y_{3} \sin \theta\right)+y_{2}^{2}+y_{3}^{2}\right) \\
& \sin \theta-\left(y_{2} \sin \theta+y_{3} \cos \theta\right)\left(2 y_{3}-2 x_{3}^{\prime} \cos \theta\right) \\
& D_{2}=\left(x_{3}^{\prime 2}-2 x_{3}^{\prime}\left(y_{2} \cos \theta-y_{3} \sin \theta\right)+y_{2}^{2}+y_{3}^{2}\right) \\
& \tau_{33}\left(y_{2}, y_{3}, \theta, t\right)=\left[\tau_{\infty}(t)-\tau_{\infty}(0) e^{-(\mu / \eta) t}\right] \sin \theta \\
& \left.+\left[H\left(t-T_{1}\right) /(2 \pi)\right] w_{1}\left(t_{1}\right) e^{(-\mu / \eta)}\right] \int_{0}^{D} \\
& g\left(x_{3}^{\prime}\right)\left[A_{3} / B_{3}+C_{3} / D_{3}\right] d x_{3}^{\prime}
\end{aligned}
$$

Where,

$$
\begin{gathered}
A_{3}=\left(x_{3}^{\prime 2}-2 x_{3}^{\prime}\left(y_{2} \cos \theta+y_{3} \sin \theta\right)+y_{2}^{2}+y_{3}^{2}\right) \\
(-\cos \theta)-\left(y_{2} \sin \theta-y_{3} \cos \theta\right)\left(2 y_{3}-2 x_{3}^{\prime} \cos \theta\right) \\
B_{3}=x_{3}^{\prime 2}-2 x_{3}^{\prime}\left(y_{3} \cos \theta+y_{3} \sin \theta\right)+y_{2}^{2}+y_{3}^{2} \\
C_{3}=\left(x_{3}^{\prime 2}-2 x_{3}^{\prime}\left(y_{2} \cos \theta-y_{3} \sin \theta\right)+y_{2}^{2}+y_{3}^{2}\right) \times \\
\times \cos \theta-\left(y_{2} \sin \theta+y_{3} \cos \theta\right)\left(2 y_{3}-2 x_{3}^{\prime} \sin \theta\right) \\
D_{3}=x_{3}^{\prime 2}-2 x_{3}^{\prime}\left(y_{2} \cos \theta-y_{3} \sin \theta\right)+y_{2}^{2}+y_{3}^{2}
\end{gathered}
$$$$
e_{23}\left(y_{2}, y_{3}, \theta, t\right)=\left(\frac{1}{2}\right)\left(e_{23}\right)_{0}+H\left(t-T_{1}\right) /(2 \pi)
$$$$
w_{1}\left(t_{1}\right) \phi_{2}\left(y_{2}, y_{3}, \theta, t\right.
$$$$
e_{33}\left(y_{2}, y_{3}, \theta, t\right)=\left(e_{33}\right)_{0}+(1 / \mu)\left[\left(\tau_{\infty}(t)-\tau_{\infty}(0)\right) /\right.
$$$$
\left.+(\mu / \eta) \int_{0}^{t} \tau_{\infty}(\tau) d \tau\right] \sin \theta+H\left(t-T_{1}\right) /(2 \times \pi)
$$$$
w_{1}\left(t_{1}\right) \phi_{3}\left(y_{2}, y_{3}, \theta, t\right)
$$

Where, $\phi_{2}\left(y_{2}, y_{3}, \theta, t\right), \phi_{3}\left(y_{2}, y_{3}, \theta, t\right)$ are given in Appendix 2. (B)

\section{Numerical Computations}

Following [1] and recent studies on rheological behavior of crust and upper mantle by[9],[14] the values of the model parameters are taken as :

$$
\begin{aligned}
& \mu=2 \times 10^{11} \text { Dynes } / \mathrm{cm}^{2} \\
& \eta=3 \times 10^{20} \text { Poise }
\end{aligned}
$$

$D=$ Depth of the fault $=10 \mathrm{~km}$., [nothing that the depth of all major earthquake faults are in between $10-15 \mathrm{~km}$ ].

$\tau_{\infty}(t)=$ constant $=\tau_{\infty}=2 \times 10^{8}$ dynes $/ \mathrm{cm}^{2}(200$ bars),[post seismic observations reveal that in most of the cases, stress released in major earthquake are of the order of 200 bars or less, in extreme cases, it may be 400 bars.]

$$
\left(\tau_{23}\right)_{0}=5 \times 10^{7} \text { Dyne } / \mathrm{cm}^{2} \text { (50 bars) }
$$$$
\text { and } \tau_{\infty}(0)=0
$$

We take the creep function $g\left(x_{3}^{\prime}\right)-W\left[\left(x_{3}^{\prime}\right)^{2}-\right.$ $\left.\left.-D^{2}\right)^{2}\right] /(D)^{4}$, with $W=1 \mathrm{~cm} /$ year, satisfying the conditions stated in $\left(C_{1}\right)-\left(C_{2}\right)$.

We now compute the following quantities:

$$
\begin{aligned}
& W_{1}\left(y_{2}, y_{3}, \theta, t\right)=w\left(y_{2}, y_{3}, \theta, t\right)-(w)_{0}-\left(y_{3} / \mu\right) \\
& {\left[\left(\tau_{\infty}(t)-\tau_{\infty}(0)\right)+(\mu / \eta) \int_{0}^{t} \tau_{\infty}(\tau) d \tau\right] \sin \theta} \\
& t_{2^{\prime} 3^{\prime}\left(y_{2} y_{3}, \theta, t\right)=\tau_{23}\left(y_{2}, y_{3}, \phi, t\right)-\left(\tau_{23}\right)_{0} e^{(-\mu / \eta) t}} t_{3^{\prime} 3^{\prime}\left(y_{2} y_{3}, \theta, t\right)=\left[\tau_{23}(t)-\tau_{\infty}(0) e^{-(\mu / \eta}\right] \sin \theta} \\
& E_{23}\left(y_{2}, y_{3}, \theta, t\right)=\left[e_{23}\left(y_{2}, y_{3}, \theta, t\right)-\left(\frac{1}{2}\right)\left(e_{23}\right)_{0}\right] \times 10^{6}(
\end{aligned}
$$




$$
\begin{aligned}
& E_{33}\left(y_{2}, y_{3}, \theta, t\right)=e_{33}\left(y_{2}, y_{3}, \theta, t\right)-\left(e_{33}\right)_{0}-\left(y_{3} / \mu\right) \\
& {\left[\left(\tau_{\infty}(t)-\tau_{\infty}(0)\right)+\left(\frac{\mu}{\eta}\right) \int_{0}^{t} \tau_{\infty}(\tau) d \tau\right] \sin \theta}
\end{aligned}
$$

where $\tau_{23}, e_{23}, e_{33}$ are given by $(\mathrm{B})$.

\section{Results and Discussion}

(A) Variation of vertical component of displacement due to creep across the fault after $t=1$ year.

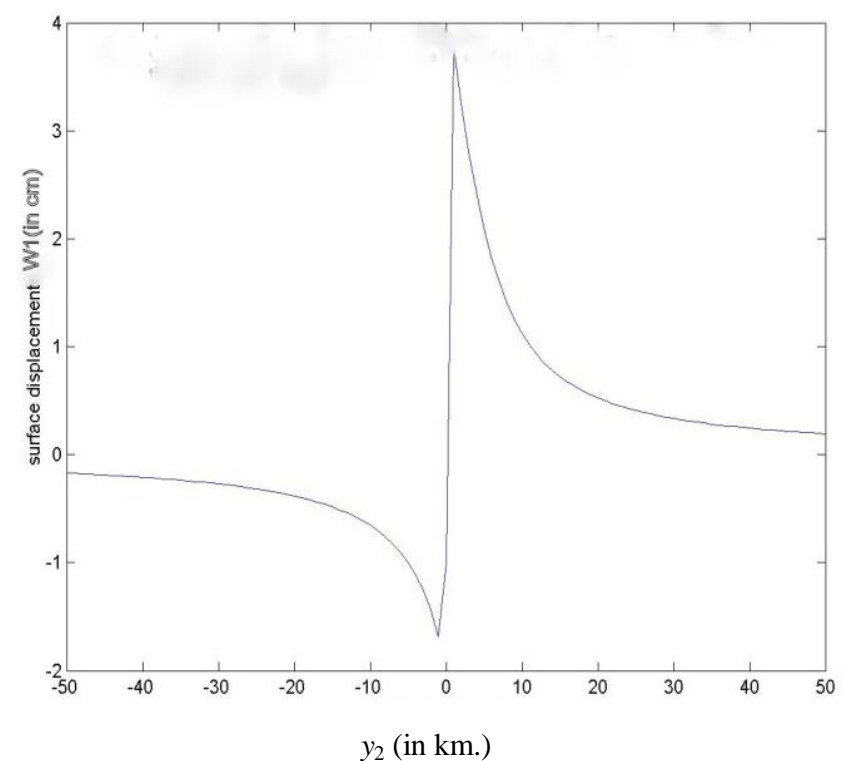

Figure 2. Variation of the vertical component of surface displacement $W_{1}$ with $y_{2}$ for $y_{3}=0, t=1$ year, $\theta=45$ (in deg) due to the fault movement

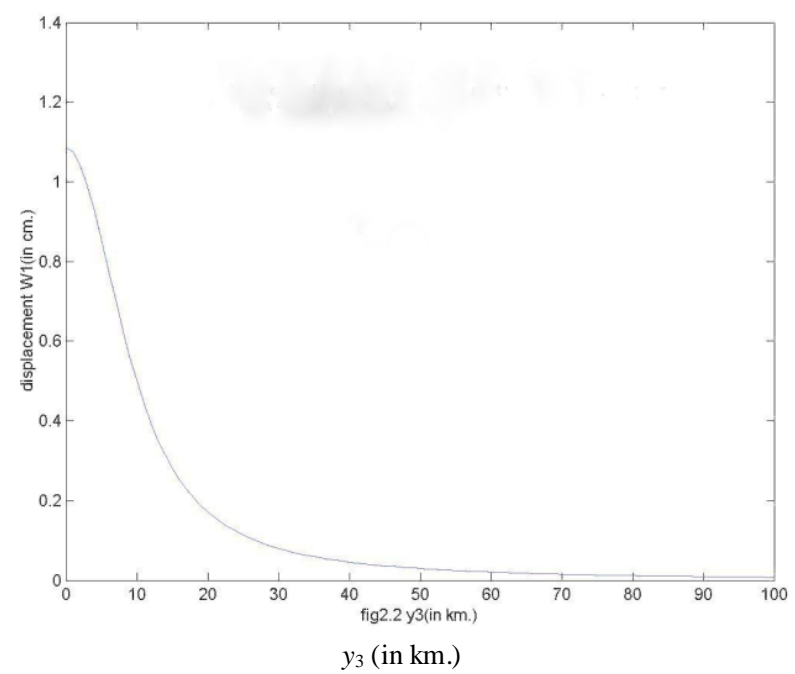

Figure 3. Variation of the vertical component of surface displacement $W_{1}$ with $y_{3}$ for $y_{2}=5 \mathrm{~km}$., $t=1$ year, $\theta=90$ (in deg) due to the fault movement

Equation (2.1) gives us the vertical component of displacement at $\left(y_{2}, y_{3}\right)$ due to the movement across the fault for different dip angle $\theta$ and at different time after the fault movement. We take $t=1$ year. In Figure 2 the graph shows the nature of surface displacement $W_{1}$ against $y_{2}$, the distance from the strike of the fault with $\theta=45$ (in deg). It is observed that the displacement are in opposite directions across the strike of the fault. Their magnitudes gradually decrease and tend to zero as we move away from the fault. This is quite expected as the effect of the fault movement gradually die out with distance. The sudden changes of $W_{1}$ near $y_{2}=0$ is due to the dip-slip motion along the fault. This is in good conformity with the results shown in[36]. Figure 3 shows the variation of $W_{1}$ with depth $y_{3}$ along the vertical through a point $y_{2}=5 \mathrm{~km}$ for a vertical fault with $\theta=90$ (in deg). It shows that $W_{1}$ decreases sharply upto a depth of about $15 \mathrm{~km}$ and thereafter dimin ishes to zero at a slower rate, and becomes significantly small for $y_{3}>100 \mathrm{~km}$.

(B) Variation with depth of the main driving stress $t_{2^{\prime}} 3^{\prime}$ in the dip-slip direction due to the movement across $F$.

Figures (4) - (7) show the variation of $t_{2^{\prime} 3^{\prime}}$ with depth $y_{3}$ for various $\theta$ and some specific values of $y_{2}$.

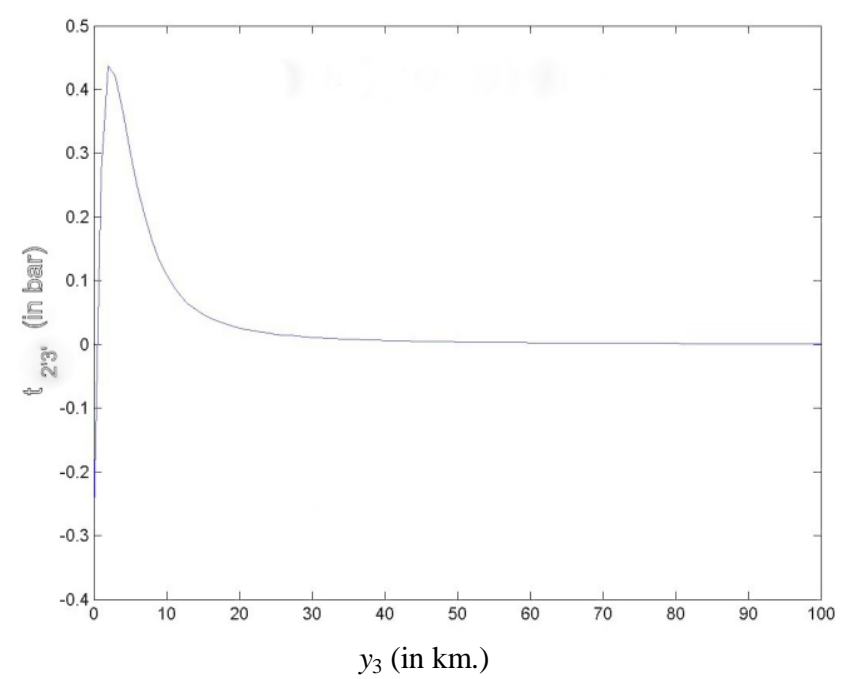

Figure 4. Variation of the stress component $t_{2} 3^{\prime}$ with $y_{3}$ for $y_{2}=1 \mathrm{~km}$., $t=1$ year, $\theta=90$ (in deg) due to the fault movement

In Figure 4, it is found that for a vertical dip-slip fault $\theta=90$ (in degree) and at a point very near to the fau lt $y_{2}=1 \mathrm{~km}$, $t_{2}{ }^{\prime} 3^{\prime}$ undergoes a change (in one year) by an amount $<0.5$ bar, due to the creeping movement across $F$. In itially there is a very small region of stress release just below the free surface $\left(0<y_{3}<1 \mathrm{~km}\right)$. Thereafter, the stress -accumulation region start, a maximum accumulation occurs at a depth of about $2 \mathrm{~km}$ below the free surface. This additional stress falls off quickly and becomes negligibly s mall at a depth of 20-25 km.

In case of the same vertical dip slip fault but a little bit away from the fault, $\left(y_{2}=5 \mathrm{~km}\right)$, the characteristic of the nature of stress accumulation and release is the similar but of magnitude of much lower order (0:04bar). Figure 6 shows the variation of main driving stress component $t_{2^{\prime} 3^{\prime}}$, with the depth for $y_{2}=5 \mathrm{~km}, t=1$ year due to the creep movement across the fault at a dip angle $\theta=60$ (degree). The graph shows that there is a negligibly s mall region $(<0.1 \mathrm{~km})$ of stress release and then it accumulates upto a depth $\geq 9 \mathrm{~km}$ and attains its maximum value 22.5 bar (approx.) for $t=1$ year and then decreases to 0 at a depth about $100 \mathrm{~km}$. 


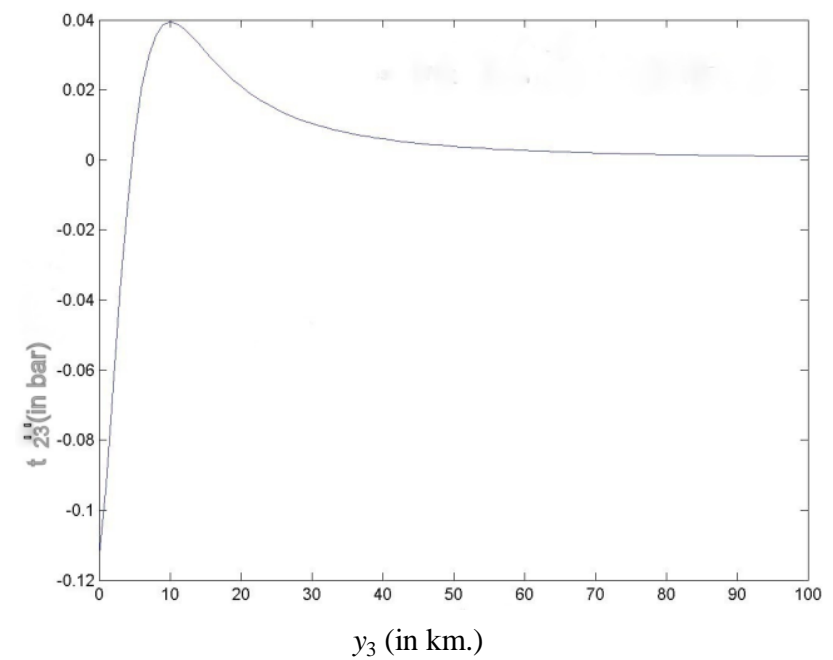

Figure 5. Variation of the stress component $t_{2} 3^{\prime}$ with $y_{3}$ for $y_{2}=5 \mathrm{~km}$., $t=1$ year, $\theta=90$ (in deg) due to the fault movement

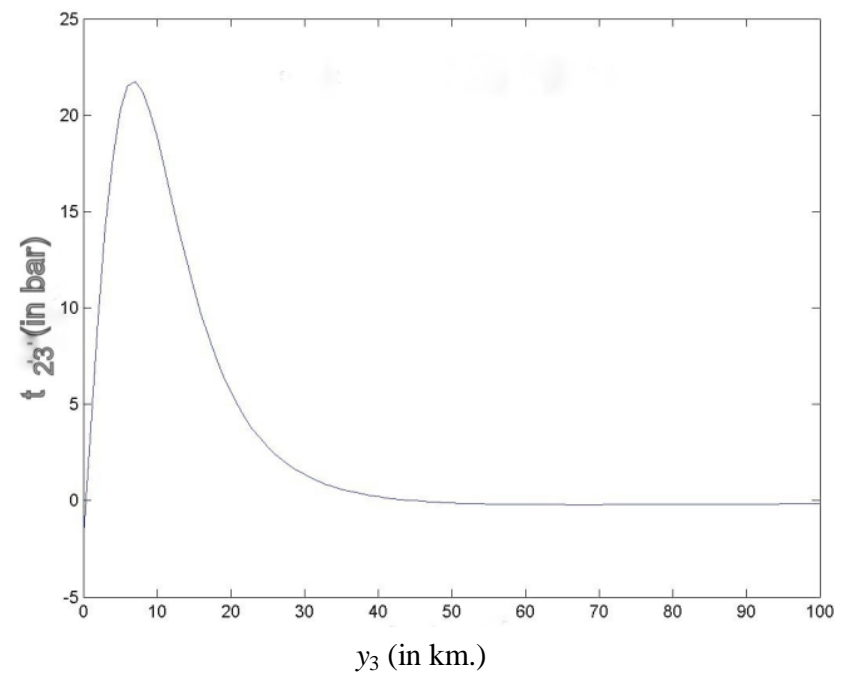

Figure 6. Variation of the stress component $t_{2}^{\prime \prime}{ }^{\prime}$ with $y_{3}$ for $y_{2}=5 \mathrm{~km}$., $t=1$ year, $\theta=60$ (in deg) due to the fault movement

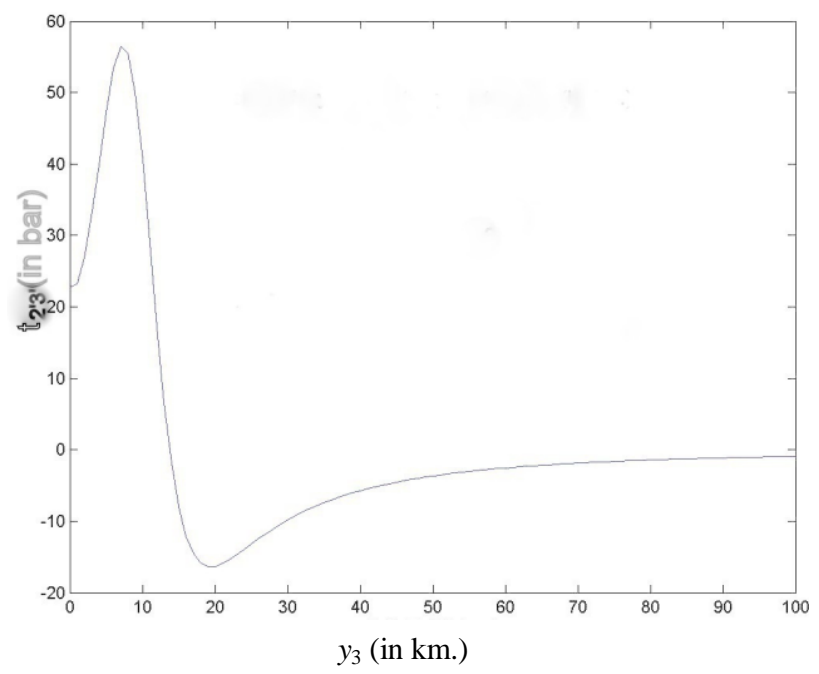

Figure 7. Variation of the stress component $t_{2} 3^{\prime}$ with $y_{3}$ for $y_{2}=9 \mathrm{~km}$., $t=1$ year, $\theta=30$ (in deg) due to the fault movement

Figure 7 shows the variation of stress component $t_{2^{\prime}} 3^{\prime}$ for $y_{2}$ $=9 \mathrm{~km}, t=1$ year and dip angle $\theta=30$ (degree) with the depth due to the creep across the fault.

We see stress accumulates in the region $0 \leq y_{3} \leq 17 \mathrm{~km}$ then it releases upto $100 \mathrm{~km}$. and become 0 at a depth about $110 \mathrm{~km}$.

Thus in the above discussion we see that due to creep movement in the dip-slip fault, there are regions where stress get released and there are certain other regions where stress accumulates. The rate of stress release/accumulation depends essentially on the dip-angle $\theta$ and the distance $y_{3}$ from the fault.

\section{ACKNOWLEDGEMENTS}

One of the authors (Subrata Kr. Debnath) thanks the Principal and Head of the Department of Basic Science and Hu manities, Meghnad Saha Institute of Technology, a unit of Techno India Group(INDIA), for allowing me to pursue the Ph.D. work, and also thanks the Geological Survey of India, Kolkata, for providing me the library facilities.

\section{Appendix-1}

Solutions for displacements, stresses and strains in the absence of any fault movement:

We take Laplace transform of all the constitutive equations and the boundary conditions (1.1)-(1.11) with respect to time and we get,

$$
\overline{\tau_{22}}=\frac{\left(p \frac{\partial \bar{v}}{\partial y_{2}}\right)-\left(\frac{\partial v}{\partial y_{2}}\right)_{0}}{\frac{1}{\eta}+\frac{p}{\mu}}+\frac{\frac{1}{\mu}\left(\tau_{22}\right)_{0}}{\frac{1}{\eta}+\frac{p}{\mu}}
$$

where, $\overline{\tau_{22}}=\int_{0}^{\infty} \tau_{22} e^{-p t} d t(p>0$, Laplace trans-formation variable) and similar other equations. Also the stress equations of motions in Laplace transform domain as:

$$
\begin{gathered}
\frac{\partial}{\partial y_{1}}\left(\overline{\tau_{11}}\right)+\frac{\partial}{\partial y_{2}}\left(\overline{\tau_{12}}\right)+\frac{\partial}{\partial y_{3}}\left(\overline{\tau_{13}}\right)=0 \\
\frac{\partial}{\partial y_{1}}\left(\overline{\tau_{21}}\right)+\frac{\partial}{\partial y_{2}}\left(\overline{\tau_{22}}\right)+\frac{\partial}{\partial y_{3}}\left(\overline{\tau_{23}}\right)=0 \\
\frac{\partial}{\partial y_{1}}\left(\overline{\tau_{31}}\right)+\frac{\partial}{\partial y_{2}}\left(\overline{\tau_{32}}\right)+\frac{\partial}{\partial y_{3}}\left(\overline{\tau_{33}}\right)=0
\end{gathered}
$$

$\overline{\tau_{22}}\left(y_{1}, y_{2}, y_{3}, p\right)=\tau_{\infty}(p) \cos \theta$ as $\left|y_{3}\right| \rightarrow \infty, y_{3}=0$ (1.7a)

On the surface $y_{3}=0,\left(-\infty<y_{2}<\infty\right)$

$$
\begin{aligned}
& \overline{\tau_{23}}\left(y_{1}, y_{2}, y_{3}, p\right)=0 \\
& \overline{\tau_{33}}\left(y_{1}, y_{2}, y_{3}, p\right)=0
\end{aligned}
$$

Also $y_{3} \rightarrow \infty\left(0 \infty<y_{2}<\infty\right)$

$$
\begin{gathered}
\overline{\tau_{23}}\left(y_{1}, y_{2}, y_{3}, p\right)=0 \\
\overline{\tau_{33}}\left(y_{1}, y_{2}, y_{3}, p\right)=\tau_{\infty}(p) \sin \theta
\end{gathered}
$$

Using (3.1), other similar equations and assuming the initial fields to be zero, we get from (1.4a), and(1.6a) 


$$
\nabla^{2}(\bar{v})=0, \nabla^{2}(\bar{w})=0
$$

Thus we are to solve the boundary value problem (3.2) with the boundary conditions (1.7a)-(1.11a).

Let,

$$
v\left(y_{2}, y_{3}, \theta, p\right)=\frac{(v)_{0}}{p}+A_{1} y_{2}+B_{1} y_{3}
$$

be the solution of (3.2)

where $A_{1}$ and $B_{1}$ are arb itrary constant which are independent of $y_{2}$ and $y_{3}$, to be determined using the in itial and boundary conditions as above.

Using the boundary conditions $(1.10 \mathrm{a})-(1.11 \mathrm{a})$ and the initial conditions we get,

$$
A_{1}=\frac{1}{p}\left[\left(\frac{1}{\eta}+\frac{p}{\mu}\right)\right]\left(\tau_{\infty}(p)-\frac{1}{P+\frac{\mu}{\eta}} \tau_{\infty}(0)\right) \sin \theta
$$

On taking inverse Laplace transformation we get,

$$
\begin{aligned}
& v\left(y_{2}, y_{3}, \theta, t\right)=(v)_{0}+\left(y_{2} \cos \theta / \mu\right) \\
& \left.\left[\tau_{\infty}(0)\right)+(\mu / \eta) \int_{0}^{t} \tau_{\infty}(\tau) d \tau\right]
\end{aligned}
$$

Similarly we can get the other components of the displacements.

$$
\begin{aligned}
& w\left(y_{2}, y_{3}, \theta, t\right)=(w)_{0}+\left(y_{2} \sin \theta / \mu\right) \\
& \left.\left[\tau_{\infty}(t)-\tau_{\infty}(0)\right)+(\mu / \eta) \int_{0}^{t} \tau_{\infty}(\tau) d \tau\right]
\end{aligned}
$$

The stresses are given by,

$$
\begin{gathered}
\tau_{32}=\left(\tau_{\infty}(t) \cos \theta-\left[\tau_{\infty}(0) \cos \theta-\left(\tau_{22}\right)_{0}\right] e^{-(\mu / \eta) t}\right. \\
\tau_{23}=\left(\tau_{23}\right)_{0} e^{-(\mu / \eta) t} \\
\tau_{33}=\left(\tau_{\infty}(t)-\tau_{\infty}(0) e^{-(\mu / \eta) t}\right) \sin \theta
\end{gathered}
$$

Using the displacements the strains can also be found out to be,

$$
\begin{gathered}
e_{22}\left(y_{2}, y_{3}, \theta, t\right)=\left(e_{22}\right)_{0} \\
e_{33}\left(y_{2}, y_{3}, \theta, t\right)=\left(\frac{1}{2}\right)\left(e_{23}\right)_{0}
\end{gathered}
$$

\section{Appendix-2}

\section{Solutions after the fault movement}

We assume that after a time $T_{1}$ the stress component $\tau_{2^{\prime} 3^{\prime}}$ (which is the main driving force for the dip-slip motion of the fault) exceeds the critical value $\tau_{c}$ and the fault $F$ starts creeping. Then we have an additional condition characterizing the dislocation in $w$ due to the creeping movement as:

$$
[(w)]_{r}=w_{1}\left(t_{1}\right) g\left(y_{1}\right) H\left(t_{1}\right)
$$

where, $H\left(t_{1}\right)$ is the Heaviside function and $[(w)]_{F}$. The discontinuity of $w$ across $F$ given by

$$
\begin{gathered}
{[(w)]_{F}=\lim _{\left(y_{2}^{\prime} \rightarrow 0+\right)}(w)-\lim _{\left(y_{2}^{\prime} \rightarrow 0-\right)}(w)} \\
\left(y_{2}^{\prime}-0,0 \leq y_{3}^{\prime} \leq D\right)
\end{gathered}
$$

Taking Laplace transformation in (4.1) we get,

$$
[(\bar{w})]_{F}=w_{1}(p) q\left(y_{3}^{\prime}\right)
$$

The fault creep commences across $F$ after time $T_{1}$, clearly, $[(w)]_{F}=0$

for $t_{1} \leq 0$, where $t_{1}=t-T_{1}, F$ is located in the region $\left(y_{3}{ }^{\prime}=0\right.$, $\left.0<y_{3}^{\prime}<D\right)$.

We try to find the solution as,

$$
\begin{gathered}
v=(v)_{1}+(v)_{2}, w=(w)_{1}+(w)_{2}, \\
\tau_{22}=\left(\tau_{22}\right)_{1}+\left(\tau_{22}\right)_{2}, \tau_{23}=\left(\tau_{23}\right)_{1}+\left(\tau_{23}\right)_{2}, \\
\tau_{33}=\left(\tau_{33}\right)_{1}+\left(\tau_{33}\right)_{2}
\end{gathered}
$$

Where $(v)_{1} ;(w)_{1} ;\left(\tau_{i j}\right)_{1}$, are continuous everywhere in the model and are given by (A). While the second part $(v)_{2} ;\left(\tau_{i j}\right)_{2}$ are obtained by solving modified boundary value problem as stated below. We note that $(v)_{2}$; is continuous even after the fault creep, so that $[(v)]_{2}=0$, while $(w)_{2}$ satis fies the dislocation condition given by (4.2).

The resulting boundary value problem can now be stated as: $(w)_{2}$ satis fies $2 \mathrm{D}$ Laplace equation as,

$$
\nabla^{2}(\bar{w})_{2}=0
$$

Where, $(\bar{w})_{2}$ is the Lap lace transformation of $(w)_{2}$, with the modified boundary condition,

$$
\begin{gathered}
\bar{\tau}_{22}\left(y_{2}, y_{3}, \theta, p\right)=0 \text { as }\left|y_{2}\right| \rightarrow \infty, y_{3} \geq 0 \\
\bar{\tau}_{23}\left(y_{2}, y_{3}, \theta, p\right)=0 \text { as } \\
y_{3} \rightarrow \infty\left(-\infty<y_{2}<\infty,\right) \\
\bar{\tau}_{33}\left(y_{2}, y_{3}, \theta, p\right)=0 \text { as } \\
y_{3} \rightarrow \infty\left(-\infty<y_{2}<\infty,\right)
\end{gathered}
$$

and the other boundary conditions are same as (1.8a)-(1.9a).

We solve the above boundary value problem by modified Green's function method following[16],[31], and the correspondence principle.

Let $Q\left(y_{2}, y_{3}\right)$ be any point in the field and $P\left(x_{2}, x_{3}\right)$ be any point in the fault, then we have,

$$
\begin{aligned}
& (\bar{w})_{2}(Q)=\int_{F}\left(w_{1}\right)(P) g\left(x_{3}^{\prime}\right)\left[G_{33}^{3}(P, Q) d x_{2}-\right. \\
& \left.-G_{32}^{3}(P, Q) d x_{3}\right]
\end{aligned}
$$

where,

$$
\begin{aligned}
& G_{33}^{3}(P, Q)=\left(\frac{1}{2 \times \pi}\right) \frac{y_{3}-x_{3}}{\left[\left(y_{2}-x_{2}\right)^{2}+\left(y_{3}-x_{3}\right)^{2}\right]}- \\
& -\frac{y_{3}+x_{3}}{\left[\left(y_{2}-x_{2}\right)^{2}+\left(y_{3}+x_{3}\right)^{2}\right]} \\
& G_{32}^{3}(P, Q)=\left(\frac{1}{2 \times \pi}\right) \frac{y_{2}-x_{2}}{\left[\left(y_{2}-x_{2}\right)^{2}+\left(y_{3}-x_{3}\right)^{2}\right]}- \\
& -\frac{y_{2}+x_{2}}{\left[\left(y_{2}-x_{2}\right)^{2}+\left(y_{3}+x_{3}\right)^{2}\right]} \\
& (\bar{w})_{2}(Q)=\int_{F}(w)_{1}(P) g\left(x_{3}^{\prime}\right) \times
\end{aligned}
$$




$$
\begin{gathered}
\frac{\left(y_{2} \sin \theta-y_{3} \cos \theta\right)}{\left[\left(x_{3}^{\prime}\right)^{2}-2 \times x_{3}^{\prime}\left(y_{2} \cos \theta-y_{3} \sin \theta\right)+\left(y_{2}\right)^{2}+\left(y_{3}\right)^{2}\right]} \\
\times d x_{3}^{\prime} \\
\left((w)_{1}(P) / 2 \times \pi\right) \phi_{1}\left(y_{2}, y_{3}, \theta\right) \text { (say) (4.9) }
\end{gathered}
$$

Where,

$$
\begin{gathered}
\phi_{1}\left(y_{2}, y_{3}, \theta\right)=\int_{F} g\left(x_{3}^{\prime}\right) \\
\frac{\left(y_{2} \sin \theta-y_{3} \cos \theta\right)}{\left[\left(x_{3}^{\prime}\right)^{2}-2 \times x_{3}^{\prime}\left(y_{2} \cos \theta-y_{3} \sin \theta\right)+\left(y_{2}\right)^{2}+\left(y_{3}\right)^{2}\right]} \\
\left.-\frac{\left(y_{2} \sin \theta+y_{3} \cos \theta\right)}{\left[\left(x_{3}^{\prime}\right)^{2}-2 \times x_{3}^{\prime}\left(y_{2} \cos \theta-y_{3} \sin \theta\right)+\left(y_{3}\right)^{2}+\left(y_{3}\right)^{2}\right]}\right] \\
\times d x_{3}^{\prime}
\end{gathered}
$$

Taking inverse Laplace transformation,

$$
(w)_{2}(Q)=w_{1}\left(t_{1}\right) \phi_{1}\left(y_{2}, y_{3}, \theta\right) H\left(t_{1}\right)
$$

Where, $H\left(t_{1}\right)$ is the Heaviside step function, which gives the displacement at any points $Q\left(y_{2}, y_{3}\right)$.

We also have,

$$
\begin{gathered}
\left(\bar{\tau}_{22}\right)_{2}=0 \\
\left(\bar{\tau}_{23}\right)_{2}=\frac{w_{1}(p)}{(2 \times \pi) \frac{p}{\frac{p}{\mu}+\frac{1}{\eta}}} \phi_{2}\left(y_{2}, y_{3}, \theta\right)
\end{gathered}
$$

where, $\phi_{2}\left(y_{2}, y_{3}, \theta\right)=\frac{\partial}{\partial y_{2}} \phi_{1}\left(y_{2}, y_{3}, \theta\right)$

$$
\left(\bar{\tau}_{33}\right)_{2}=\frac{w_{1}(p)}{(2 \times \pi)} \frac{p}{\frac{p}{\mu}+\frac{1}{\eta}} \phi_{3}\left(y_{2}, y_{3}, \theta\right)
$$

where, $\phi_{3}\left(y_{2}, y_{3}, \theta\right)=\frac{\partial}{\partial y_{3}} \phi_{1}\left(y_{2}, y_{3}, \theta\right)$

\section{REFERENCES}

[1] Aki. K, and Richards. P.G : Quantitative Seismology: Theory and Methods; W.H. Freeman, San Francisco, California, 1980.

[2] Ben-Menahem. A, Gillon. A : Crustal deformation by earthquakes and explosions. Bulletin of the seismological society of America.vol-60,no-1,pp193-323 215, 1971.

[3] Blake.T.R, Dienes. J.K (1976) On viscosity and the inelastic nature of waves in geological media, B.S.S.A Vol-66,no-2, pp-453-465, 1976.

[4] Chinnery. M.A : The deformation of the ground arround surface faults; Bull. Seis.Soc. Am,51,355-372, 1961.

[5] Chinnery.M.A : The strength of the Earth's crust under horizontal shear stress; Jour.Geophy sical.Res.69.2085-2089, 1964.

[6] Chinnery. M.A : The vertical displacements associated with transcurrent faulting; J.Geophys.Res.70,4627-4632, 1965.

[7] Chinnery.M.A and Dushan. B. Jovanovich: Effect of the earth's layering on earthquake displacement field; Bull. Seis. Soc. Am, 62, 1629-1646, 1972.

[8] Cohen : Post seismic viscoelastic surface deformations and stress, 1, Theoretical considerations, Displacements and strains calculations, J.Geophys.Res. 85, No. B6, 3131-3150, 1980a.

[9] Chift. P, Lin. J, Barcktiausen. U: Marine and Petroleum Geology 19,951-970, 2002.

[10] Fuis. S.G, Scheirer S.D, Langenheim. E.V, Kohler. D.M : A new perspective on the geometry of the San Andreas fault of south California and iots, relationship to lithospheric structure, B.S.S.A vol-102,pp236-1251, 2012.

[11] Freaund. L.B, Barnett. D.M : A two-dimensional analy sis of surface deformation due to dip-slip faulting, B.S.S.A, vol-66, No-3, pp-667-675, 1976.

[12] Ghosh, Mukhopadhy ay. A and Sen. S: On two interacting creeping vertical surface-breaking strike-slip faults in a two-layered model of lithosphere. Physics of the Earth and planetary interior.70, 119-129, 1992.

[13] Ghosh. U. and Sen. S : Stress accumulation near buried fault in lithosphere-asthenosphere sy stem; International Journal of computing,Oct,2011,vol-I,Issue 4,pp 786-795, 2011.

[14] Karato. Shun-Ichiro : Rheology of the Earth's mantle: A historical review Gondwana Research-Vol.18-issue-1,pp 17-45, 2010.

[15] Maruy ama. T. : Statical elastic dislocations in an infinite and semi-infinite medium; Bull. Earthquake. Res. Inst., Tokyo Univ., 42, 289-368, 1964.

[16] Maruy ama. T.: On two dimensional dislocations in an infinite and semi-inf inite medium; Bull. Earthquake Res. Inst. Tokyo Univ.,44,(part 3).p.811-871, 1966.

[17] Matsu'ura. M, Sato. R : Static deformation due to fault spreading over several layers in multi-layered medium part-II-Strain and tilt; Journal of the Phys. of the Earth, vol-23,no-1,12-33, 1975.

[18] Mukhopadhyay. A et al : On stress accumulation near finite rectangular fault,Indian journal of Meteorology, Hydrology and Geophy sics (Mausam), vol-30, pp-347-352, 1979a.

[19] Mukhopadhy ay. A et al : On stress accumu lation and fault slip in lithosphere, Indian journal of Meteorology, Hydrology and Geophy sics (Mausam), vol-30, pp-353-358, 1979 b.

[20] Mukhopadhyay.A,Sen.S and Paul.B.P: On stress accumulation in a viscoelastic lithosphere containing a continuously slipping fault; Bull. Soc. Earthquake Technology,vol-17,No-1, pp-1-10, 1980,a.

[21] Mukhopadhyay. A, Sen. S, and Paul. B.P : On stress accumulation near a continuously slipping fault in a two layered model of lithosphere; Bull. Soc. Earthquake Technology, vol-17, No-4,p-29-38, 1980a.

[22] Matsu'ura. M, Sato. R : Static deformation due to fault spreading over several layers in multi-layered medium part-I-Strain and tilt; jour. of the phys. of the Earth, vol. 23,no-1,12-33, 1975.

[23] Mansinha. L, Smylie. D.E : The Displacement fields of inclined faults; Bulletin of the seismological society of America, vol. 61, no 5, 1433-1440. 1971. 
[24] Nur. A, Mavko. G : Post-seismic viscoelastic rebound,Science,183,204-206, 1974.

[25] Oglesby. D.D : The Dynamics of Strike-slip Step-overs with linking Dip-slip faults, B.S.S.A,vol-95, No.-5, PP-1604-1622, 2005.

[26] Okada. Y : Internal deformation due to shear and tensile faults in a half-space, B.S.S.A. vol-82, No.2. pp.1018-1040, 1992.

[27] Press. F : Displacements, Strains and Tilts at teleseismic Distances, Journal of Geophysical Research, vol 70, no-10, 1965.

[28] Rani. S and Singh. S.J : Static deformation of a uniform half space due to a long dip-slip fault. Geophy. J. Int. vol. 109, pp 469-476, 1992.

[29] Rundle. J.B: Viscoelastic crustal deformation by finite quasi-static sources, Journal of the Geophysical Research, vol-83, no-B12, pp-5937-5945, 1978.

[30] Rudnicki. J.W, WU. M : Mechanics of dip-slip faulting in an elastic half-space, Journal of the Geophysical Research, vol-100, no-B11, PP-22, 173-22, 186, 1995.

[31] Rybicki. K. : The elastic residual field of a very long strike slip fault in the presence of a discontinuity; Bull. Seis. Soc. Am. vol.61,79-92, 1971

[32] Sato. R. : Stress drop of finite fau lt; J. Phy s Earth, 20, 397-407, 1972.

[33] Sato. R, Yamashita. T. : Static deformations in an obliquely layered medium Part-ii dip-slip fault, Journal of the phys. of the Earth 23,113-125, 1975.

[34] Savage. J.C : Displacement field for an edge dislocation in layered half space, Journal of Geophy sical Research vol-103, no-B2 pp 2439-2446, 1998.

[35] Savage. J.C and Hastie. L.M : Surface deformation associated with dip-slip faulting: J.G.R71,NO:20 4897-4904, 1966.

[36] Segal. P. : Earthquake and volcano deformation : Princeton University Press, 2010.

[37] Steketee. J.A. : On Volterra's dislocations in a semi- infinite medium; Can. J. Phys. 36. 192-205, 1958,a.

[38] Steketee. J.A. : Some geophysical applications of the theory of dislocations, Can.J.Phy s.36.1168-1198, 1958,b.

[39] Spence. D.A and Turcotte. D.L : An elastostatic deformation of a uniform half space due to a long dip-slip fault.Geophy.j.Int.Vol.109, PP-469-476, 1976.

[40] Sen. S, Sarker. S and Mukhopadhyay. A : A Creeping and surface breaking long strike -slip fault inclined to the vertical in a viscoelastic half space, Mausam, 44, 4, 365-4, 372, 1993.

[41] Singh. S.J, Rosenman. M : Quasi static deformation of a viscoelastic half-space by a displacement dislocation, Phys. of the Earth and planetary interiors, 8, 87-101, 1974.

[42] Singh. S.J, Punia. M, Kumari. G : Deformation of a layered half-space due to a very long dip-slip fault. Proc. Indian Natn. Sci.Acad.63A,NO-3,PP-225-240, 1997.

[43] Tomar. S.K, Dhiman : 2D-Deformation analysis of a half-space due to a very long dip-slip fault at finite depth-Indian Acad. Sci.(Earth. Planet, Sci.)112, no-4, pp-587-596, 2003.

[44] Yu-Ting-To, Rundle. J.B, Fernandez. J: Deformation produced by a rectangular dipping fault in a viscoelastic gravitational layered Earth model part-ii: strike-slip fault-strgrv and strgrh fortran programs, Computers and Geo Sciences vol=22,no-7, pp-751-764, 1996.

[45] Zhang. C, Oglesby. D.D, Xu. G: Earthquake nucleation on dip-slip faults with depth-dependent frictional properties, Journal of Geophysical Research, vol-111, no-10, B07303, 2006. 